\title{
The Evaluation of Surface Diffusion Coefficients of Gold and Platinum Atoms at Electrochemical Interfaces from Combined STM-SEM Imaging and Electrochemical Techniques
}

\author{
C. Alonso, R. C. Salvarezza, ${ }^{1}$ J. M. Vara, and A. J. Arvia ${ }^{1, *}$ \\ Departamento de Quimica, Universidad Autónoma de Madrid, 28049 Madrid, Spain \\ L. Vazquez, A. Bartolome, and A. M. Baro* \\ Departamento de Fisica de la Materia Condensada, Universidad Autónoma de Madrid, 28049, Madrid, Spain
}

\begin{abstract}
A simple method is presented for measuring the surface diffusion coefficients of $\mathrm{Au}$ and $\mathrm{Pt}$ atoms at electrodispersed electrodes of the same metals in contact with $0.5 \mathrm{M} \mathrm{H}_{2} \mathrm{SO}_{4}$. The technique is based upon the time dependence of the surface roughness factor of electrodispersed metal overlayers. The method requires a model for the surface roughness of the metal structure. The model is deduced from microscopic measurements by a STM integrated into a conventional SEM microscope. This allows the relationship between the roughness factor and the area of the surface structure to be obtained. For $\mathrm{Au}$ and $\mathrm{Pt}$ in contact with an electrolyte solution, the values of our diffusion coefficients are higher than those reported in vacuum at the same temperature.
\end{abstract}

The evaluation of surface diffusion coefficients from particle coalescence processes is widely used in solid-state physics. Usually the methods involve the estimation of the time dependence of crystal size by microscopy (1), optical diffraction (2), or ion scattering (3), and the use of the theory developed first by Mullins (4) and extended in recent years by several authors $(5,6)$.

Surface diffusion plays an important role in many electrochemical processes such as electrocrystallization of metals, metallic corrosion, and electrocatalysis. Typical examples of materials whose properties become time dependent due to surface processes, involving the surface diffusion of metal atoms, include the electrochemical behavior (7) and structure (8) of electrodeposits grown under different conditions, the stability of small metallic particles used in dispersed-type metal electrodes as those used for electrochemical energy conversion devices (9), and the surface roughness properties of large active area electrocatalysts (10). Atomic mobility, increased by surface contaminants, also appears to be involved in stress corrosion cracking (11).

In spite of the importance of surface diffusion, available diffusion coefficients as determined in vacuum (1) or in air do not account for the presence of the electrolyte.

We have recently undertaken a study of the roughness structure of samples produced by an electro-oxidationelectrodeposition process (electrodispersed metal overlayers) (7). The combination of electrochemical measurements together with microscopic measurements by a scanning tunneling microscope (STM) integrated into a conventional SEM (16) has resulted in a model for the roughness structure. This model attributes the large roughness factor of the electrodes to the area of columnar type grains (Fig. 1). The lateral area along the depth of the reduced metal is responsible for the roughness increase. The radius of columns can be measured by STM. The height can be related to both electrochemical and SEM data (7). However, the columnar structure is unstable, decreasing its surface area and, accordingly, the surface roughness factor with the aging time.

We propose, in the present paper, a simple method to estimate the surface diffusion coefficients of metal atoms in electrochemical systems based on the time dependence of the surface roughness factor of electrodispersed metal overlayers as measured by conventional voltammetry. The technique proved to be successful for evaluating the surface diffusion coefficients of $\mathrm{Au}$ and $\mathrm{Pt}$ atoms in contact with aqueous acid solutions at different temperatures. The

* Electrochemical Society Active Member.

1 Permanent address: INIFTA, Universidad Nacional de la Plata, Casilla de Correo 16, Sucursal 4, 1900 La Plata, Argentina. results are compared with literature values for those metals in vacuum and air.

\section{Experimental}

The electrochemical arrangement consisted of a threecompartment glass cell provided with a polycrystalline (pc) wire working electrode (Au 99.999\% and Pt 99.999\%), a large counterelectrode of the same metal used as working electrode and a mercury/mercurous sulfate reference electrode in solution of $0.5 \mathrm{M} \mathrm{H} \mathrm{H}_{2} \mathrm{SO}_{4}$ deaerated by bubbling purified $\mathrm{N}_{2}$. In the text, the working electrode is referred to as the standard hydrogen electrode (SHE).

The experimental work comprised the following stages:

The preparation of the electrodispersed metal electrode.-The procedure for the preparation of the electrodispersed metal involved, first, the accumulation of a relatively thick metal oxide layer on the metal electrode and, second, its electroreduction to produce the electrodispersed metal overlayer (12). The relatively thick oxide layer accumulation resulted from the application of repetitive square wave perturbing potential (RSWPP) to the

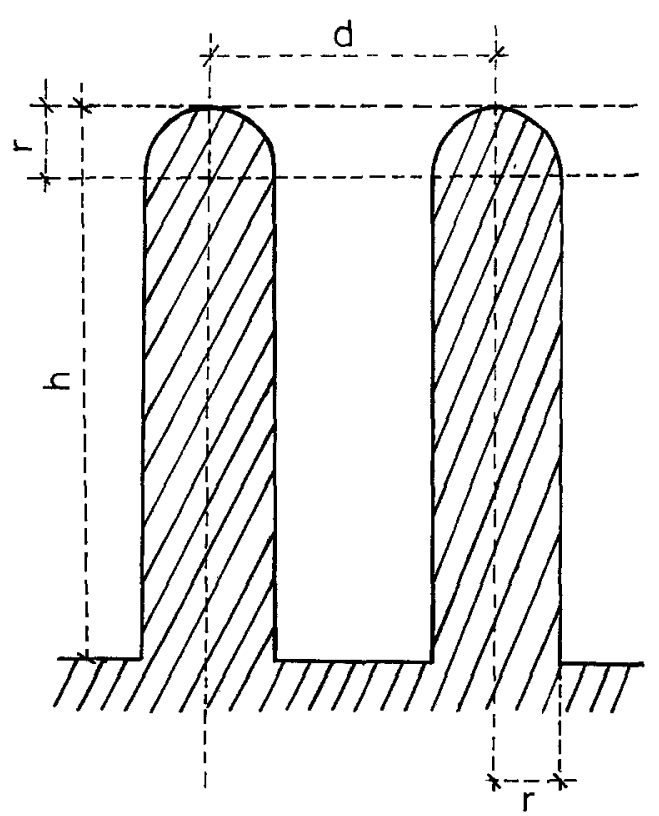

Fig. 1. Cross section of an ideal rounded cop columnar structure. The column height, $h$, and radius $r$ are indicated. The column radius is equal to the rounded cap radius. $d$ is the intercolumn distance. 
working electrode at a frequency of $4 \mathrm{kHz}$, the lower and the upper potentials being fixed at 0.6 and $3.1 \mathrm{~V}$ for $\mathrm{Au}$ and 0.05 and $2.0 \mathrm{~V}$ for Pt.

The metal overlayers were obtained through the potentiodynamic electroreduction of the thick oxides layers proceeded at a potential sweep rate of $0.02 \mathrm{~V} / \mathrm{s}$ for Au oxide and $0.001 \mathrm{~V} / \mathrm{s}$ for Pt oxide-covered electrodes. Under these conditions, the RSWPP procedure yielded the maximum development of roughness for electroreduced $\mathrm{Au}$ and $\mathrm{Pt}$ overlayers, respectively $(13,14)$. The corresponding electroreduction charge density, $q$, was evaluated from the potentiodynamic electroreduction profiles.

Electrochemical evaluation of the metal overlayer rough ness factor.-The surface roughness factor of the metal overlayer, $R$, was defined as the ratio between the $O$ adatom monolayer electrodesorption charge density determined voltammetrically for preset potential, sweep rate, and switching potential conditions for the metal overlayer and the charge density for the O-adatom monolayer on both polycrystalline $\mathrm{Au}$ and $\mathrm{Pt}$. The value of the latter for both metals was taken as $0.42 \mathrm{mC} / \mathrm{cm}^{2}(15)$.

Open-circuit aging of the metal overlayer.-The metal overlayers were aged at open circuit by keeping them continuously immersed in the deaerated $0.5 \mathrm{M} \mathrm{H}_{2} \mathrm{SO}_{4}$ and at a constant temperature, T. Periodically, the value of $R$ of the decaying specimens was tested.

SEM and STM ex situ imaging.-The ex situ SEM-STM images of the different specimens were obtained immediately after each specimen was removed from the electrochemical cell by using the combined SEM-STM microscope. This instrument allows one to image simultaneously a preselected domain of the electrode surface by means of both microscopes. A detailed description of this microscope, and its application have been reported elsewhere $(16,17)$. To obtain the STM images special care was taken to select the tip. For these highly corrugated specimens, the macroscopic shape of the tip becomes extremely important to obtain a good quality image (17). We used $\mathrm{W}$ tips electrochemically etched in $0.1 \mathrm{~N} \mathrm{NaOH}$. We obtained tips of typically $0.1 \mu \mathrm{m}$ radius as we could actually measure by SEM (7).

The following imaging procedure was applied. First, the SEM device was used to address the tip to a representative region of the specimen, and the STM images were taken. The sampled region was identified and the corresponding
SEM micrographs were obtained. The STM data are displayed as three-dimensional representations.

\section{Results}

Kinetics of the aging process.-The time evolution of $R$ for electrodispersed $\mathrm{Au}$ and $\mathrm{Pt}$ overlayers in $0.5 \mathrm{M} \mathrm{H}_{2} \mathrm{SO}_{4}$ under open circuit was followed through a voltammetry run at $0.1 \mathrm{~V} / \mathrm{s}$ between 0.05 and $1.60 \mathrm{~V}$. For both $\mathrm{Au}$ and $\mathrm{Pt}$ overlayers $R$ decreases as $t$, the aging time, increases (Fig. 1a and b). The initial rate of change of $R[(d R / d t) t \rightarrow 0]$ becomes considerably greater as $T$ is raised from 273 to $325 \mathrm{~K}$ (Fig. 2a). At constant $T,(d R / d t) t \rightarrow 0$ increases according to the volume of the electrodeposited metal overlayer proportional to $q$ (Fig. 2b), which in turn depends on the accumulated oxide layer average thickness. The latter can be adjusted by changing the duration of the RSWPP treatment to build up the thick oxide layer on the electrode surface.

For a constant value of $q$ and $t>300 \mathrm{~s}$, the dependence of $R$ on $t$ can be deduced from the linear behavior of $1 / R v s$. $t^{1 / 4}$. The slopes increase when $T$ increases (Fig. 3a). Besides, at a constant $T$, the values of the slopes decrease as $q$ increases (Fig. $3 b$, c) but under comparable experimental conditions the rate of change of $R$ with $t$ for the Au overlayer is considerable larger than for the $\mathrm{Pt}$ overlayer.

SEM and STM observations.-SEM observations of cross sections of $\mathrm{Au}\left(q=1.2 \mathrm{C} / \mathrm{cm}^{2}\right)$ and $\mathrm{Pt}\left(q=0.7 \mathrm{C} / \mathrm{cm}^{2}\right)$ overlayers immediately after their preparation indicate that the average values of the metal overlayer thickness, $h$, is $h \approx 9 \times 10^{-5}$ and $h \approx 4 \times 10^{-5} \mathrm{~cm}$, respectively (Fig. 4a and b). These figures of $h$ do not change appreciably along the aging process $\left(t=9 \times 10^{4} \mathrm{~s}\right)$.

The surface structure of the electrodispersed $\mathrm{Au}$ and $\mathrm{Pt}$ electrodes as observed by STM (Fig. 5a, 6a) shows the development of grains. They can be described as a rolling hill structure. The corrugation normal to the surface is very variable. This has two consequences:

1. For high corrugation values, tip size influences the slope of the structures, but the latter is independent of the STM scan speed; thus, the measurement of the radius is more precise on the low-corrugation grains.

2. Contour-line representation, which is useful to get a better image of grain slope, loses resolution in the $z$-direction (this is the reason why we do not show such representation of the data).
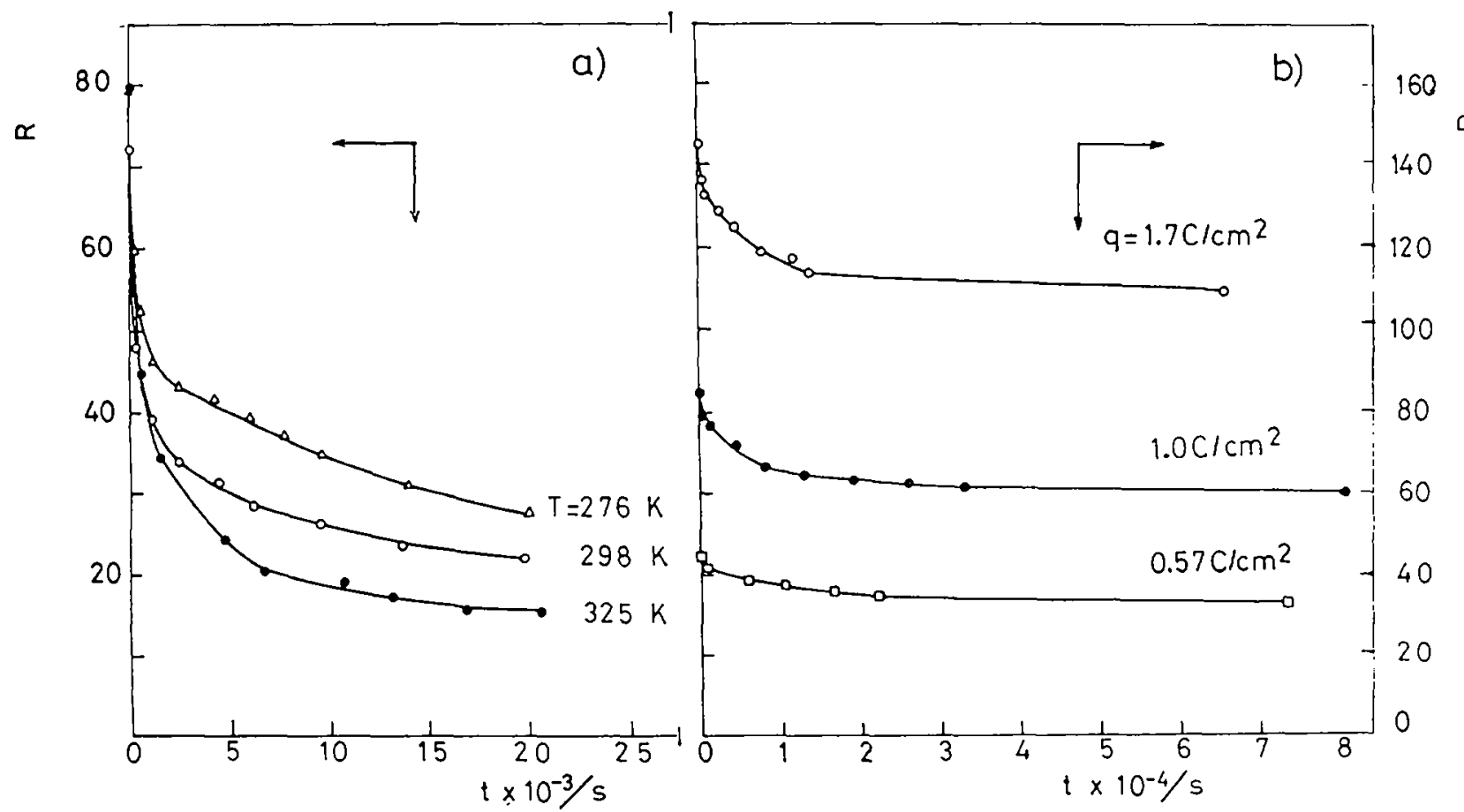

Fig. 2. $R$ vs. $t$ plots. (a) Electrodispersed Au overloyers produced from o constont amount of gold oxide, $q=1.9 \mathrm{C} / \mathrm{cm}^{2}$. Temperoture influence. (b) 


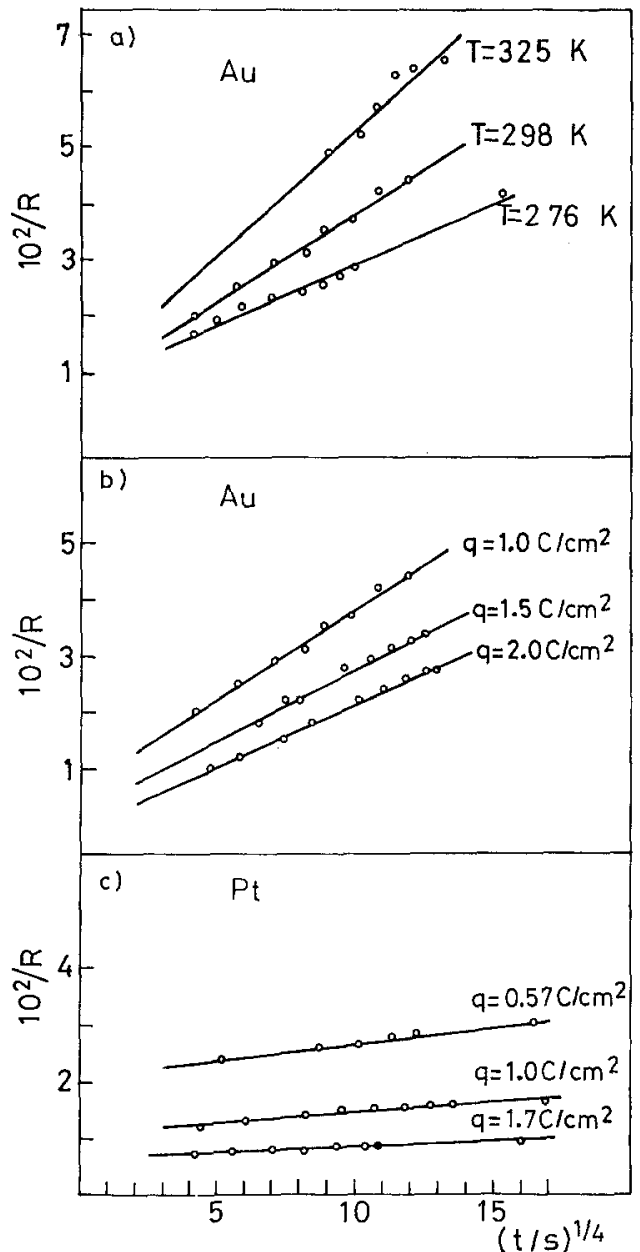

Fig. 3. $1 / R$ vs. $t^{1 / 4}$ plots. (a) Data from Fig. 1a. (b) Electrodispersed Au formed from different amounts of gold oxide, $T=298 \mathrm{~K}$. (c) Dato from Fig. 1b.

With these ideas in mind we have estimated an average radius of the grains of $\sim 150 \AA$ for Au electrodes and $\sim 100 \AA$ for Pt. So, the grains in Pt are smaller than in $\mathrm{Au}$.

The aging process is characterized by a coalescence of grains. This process is better observed on $\mathrm{Au}$, as it can be even observed by SEM. Figure $5 \mathrm{~b}$ is a three-dimensional representation of STM data corresponding to Au. Three points have to be considered:

1. The size of the aged structures formed by small units can reach a radius $\sim 1000 \AA$

2. The individual units which form the big grains are resolved in the STM image. They are grouped into four or more units.

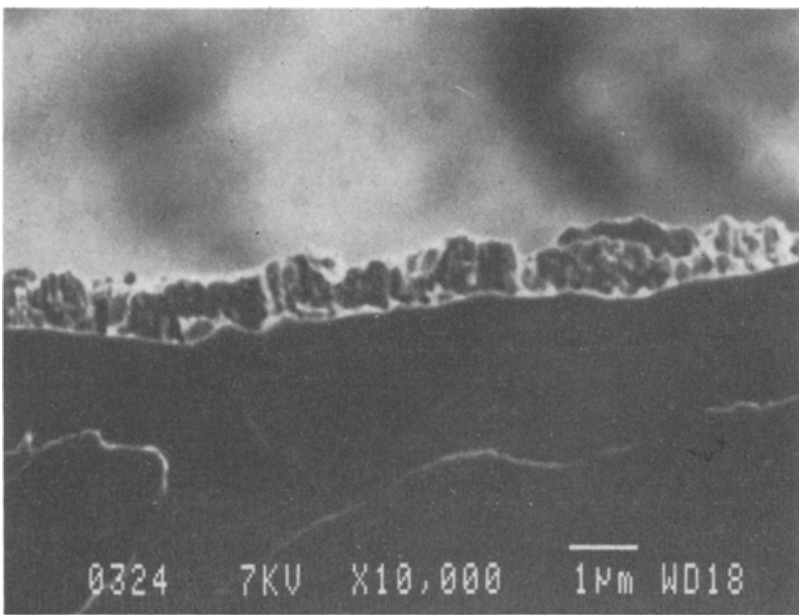

3. Big channels left after the process are observed in the STM image. The channels must develop because the coalescence process occurs without a change in the depth of the metallic layer.

Pt relaxation, on the other hand, is not so acute but morphological changes are clearly observed in the STM image (Fig. 6b): the grains are smoother, bigger, and more uniform. It is important to note that for Pt the process of coalescence is only resolved in our STM/SEM combination by the STM. Another important result is the clear link between the roughness factor $R$ and the average radius of the grains $r$ observed by STM. This fact was seen previously for $\mathrm{Au}(7)$ and is now confirmed for $\mathrm{Pt}$.

\section{Discussion}

For an ensemble of small growing particles in order to minimize the total surface and interfacial free energy, $\gamma$, of the system, the coalescence process is thought to proceed by surface diffusion of adatoms from sites of higher to sites of lower chemical potential, i.e., a surface diffusion process from small to large size particles. This mechanism implies that concentration gradients of surface adatoms originate at the crystallites surfaces (4), and in this case the time dependence of the radius of the coalescing particles, $r$, is given by the expression

$$
\frac{d\left(r^{4}\right)}{d t}=\frac{2 \gamma a^{4} D_{s}}{k T}
$$

where $a$ is the lattice parameter, $D_{\mathrm{s}}$ is the surface diffusion coefficient, $\gamma$ is the surface tension, and $k$ is the Boltzmann constant. The integration of Eq. [1] between $t=0$ and $t$ results

$$
r^{4}-r_{\mathrm{o}}^{4}=\frac{2 \gamma a^{4} D_{\mathrm{s}} t}{k T}
$$

where $r_{0}$ is the radius for $t=0$.

For the condition

$$
\frac{2 \gamma a^{4} D_{\mathrm{s}} t}{k T}>>r_{\mathrm{o}}^{4}
$$

Eq. [2] reduces to

$$
r^{4}=\frac{2 \gamma a^{4} D_{\mathrm{s}} t}{k T}
$$

Equation [3] implies that for a certain range of $t$ satisfying the above mentioned condition a linear $r v s . t^{1 / 4}$ relationship is fulfilled. Therefore, Eq. [2] and [4] allow the value of $D_{s}$ to be obtained through the measurement of the change in $r$ with time either by microscopy (1) or by diffraction (2) techniques. This way of evaluating $r$ is not easily applicable to electrochemical systems, particularly those involving large surface areas of electrodes and crystallite sizes in

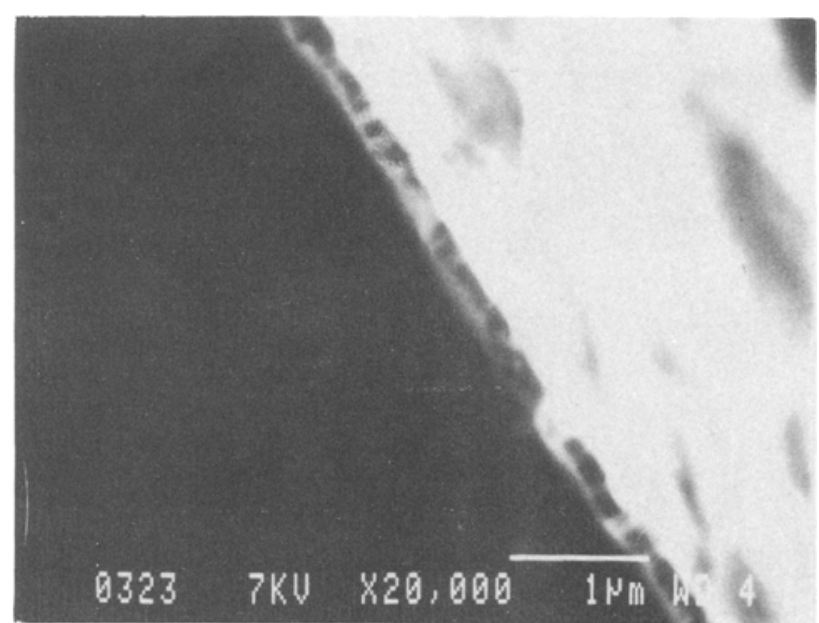

Fig. 4. SEM micrographs of the electrodispersed metal overlayer cross sections: ( $a$, left) Au; (b, right) Pt 

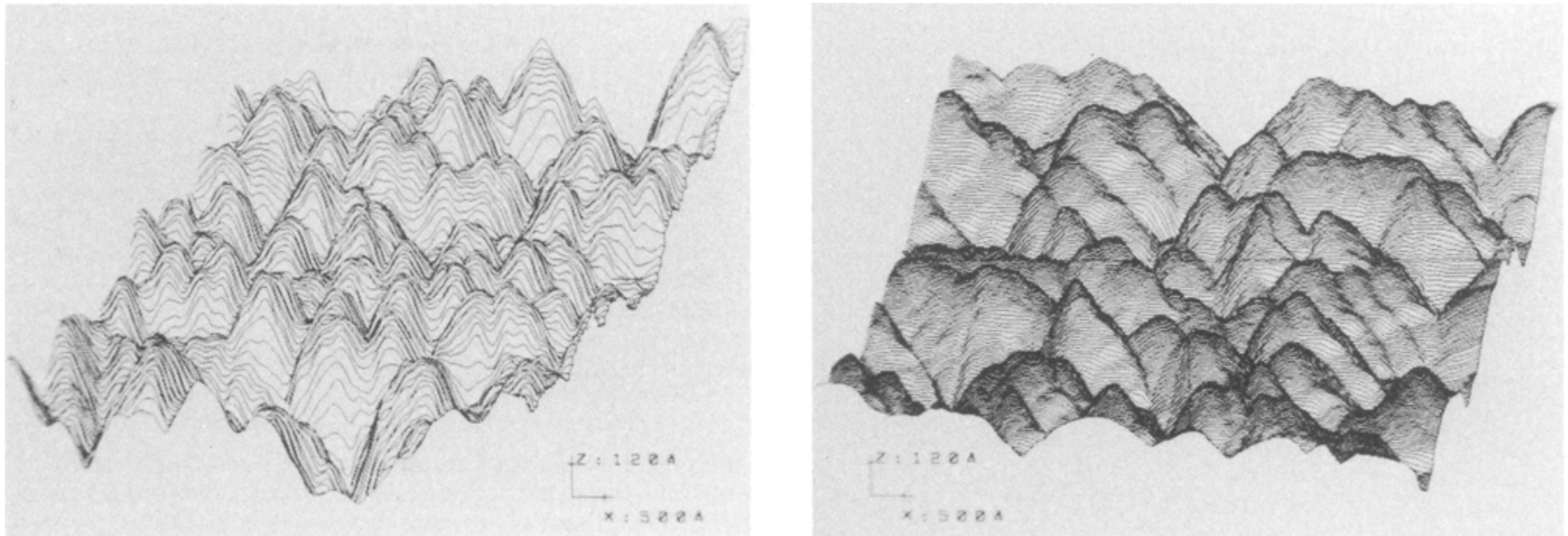

Fig. 5. (a, left) STM of electrodispersed Au obtained immediately after its formation; (b, right) the same specimen after $6 \times 10^{4} \mathrm{~s} a g i n g$ in $\mathrm{H}_{2} \mathrm{SO}_{4}$ at $325 \mathrm{~K}$. The total area imaged is $0.78 \times 0.58 \mu \mathrm{m}$.

the order of nm. As shown in Fig. 2 and 3 and in STM image (Fig. 5, 6), the time dependence of $R$ as resulting from conventional voltammetry, appears to be directly related to changes in the average particle size. This fact offers then the possibility of estimating the value of $D_{\mathrm{s}}$ from the time dependence of $R$ which reflects the average particle size of the electrode material.

For both $\mathrm{Au}$ and $\mathrm{Pt}$ electrodispersed overlayers, the combined STM/SEM imaging conclusively indicates the development of a rounded cap columnar-type structure. In the case of a close-packed array of columns, the relationship among $R$ the average column height, $h$ and its average radius, $r$, for $h \gg r$ is given by the following expression (7)

$$
R(t)=\frac{\pi h(t)}{2 r(t)}
$$

where $R(t), h(t)$, and $r(t)$ denote the instantaneous values of $R, h$, and $r$, respectively.

The time dependence of $r$ is given by Eq. [2] and [4]. Otherwise, let us assume that the aging process involves a rate of change of $r$ much greater than that of $h$, i.e., the condition $(d h / d t) / h<<(d r / d t) / r$ is fulfilled. Then it results

$$
h \simeq h_{\mathrm{o}}
$$

where $h_{0}$ denotes the $h$ value for $t=0$.

The validity of Eq. [5] is supported by SEM micrographs of the overlayer cross section exhibiting after aging no appreciable changes of $h$. Besides, it is also reasonable to consider that the value of $h$ is directly related to $h(o x)$, the oxide layer average thickness accumulated during the RSWPP treatment. Thereby, the value of $h(o x)$ can be estimated from the equation

$$
h(\mathrm{ox})=\frac{M q}{z \mathbf{F} \rho}
$$

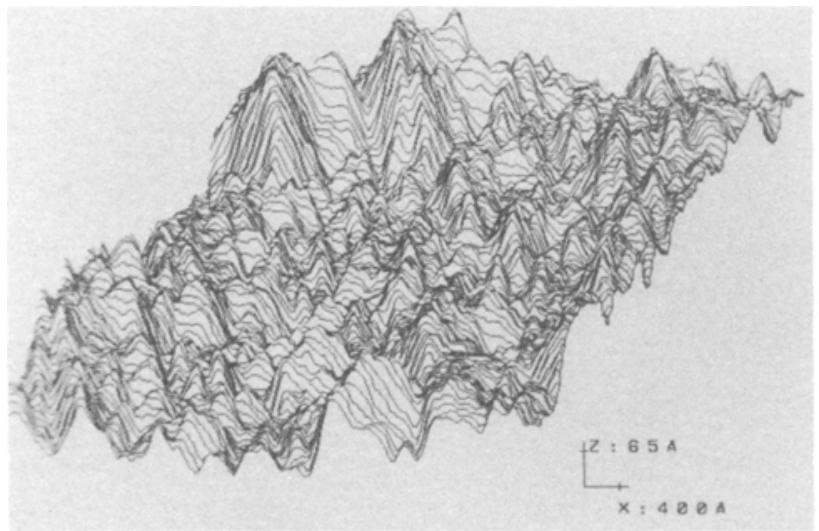

where $M$ and $\rho$ are the molecular weight and the density of the oxide, respectively. $\mathbf{F}$ is the Faraday constant, $z$ is the number of electrons transferred per mole of oxide, and $q$ is the corresponding electroreduction charge density. By using Eq. [7] with the following figures $M=442 \mathrm{~g} / \mathrm{mol}$, $\rho=11 \mathrm{~g} / \mathrm{cm}^{3}, \quad z=6, \quad q=1.2 \mathrm{C} / \mathrm{cm}^{2}$ for $\mathrm{Au}_{2} \mathrm{O}_{3}$ and $M=227 \mathrm{~g} / \mathrm{mol}, \rho=10.2 \mathrm{~g} / \mathrm{cm}^{3}, z=4, q=0.7 \mathrm{C} / \mathrm{cm}^{2}$ for $\mathrm{PtO}_{2}$, the resulting values of $h(\mathrm{ox})$ are $8 \times 10^{-5}$ and $4 \times 10^{-5} \mathrm{~cm}$ for $\mathrm{Au}$ and $\mathrm{Pt}$, respectively. These figures are in good agreement with the values of $h$ estimated from the corresponding metal overlayer cross sections (Fig. 4). These results demonstrate that the electroreduction of the metal oxide layer yielding the electrodispersed metal under the experimental conditions described in the present work, is a constant volume process so that a low density metal layer is produced. Accordingly, the following approximation can be written

$$
h=h(\mathrm{ox})
$$

i.e., the height of the columnar structure can be taken approximately equal to the height of the oxide layer. This conclusion is consistent with the structure of the overlayer as derived from the STM-SEM data consisting of a columnar structure and a large number of voids and channels. So, the Eq. [5] valid for a close-packed cubic array has to be changed to take into account the lower density of the deposit and the fact that $h \approx h(0 x)$. This can be done just by including a factor $2 / 3$ in Eq. [3], so [5] becomes

$$
R(t)=\frac{\pi h(t)}{3 r(t)}
$$

Combining Eq. [4], [6], [7], [8], and [9] one obtains

$$
R(t)=\frac{\pi M q}{3\left(2 \gamma a^{4} D_{s} / k T\right)^{1 / 4}(z \mathbf{F} \rho) t^{1 / 4}}
$$

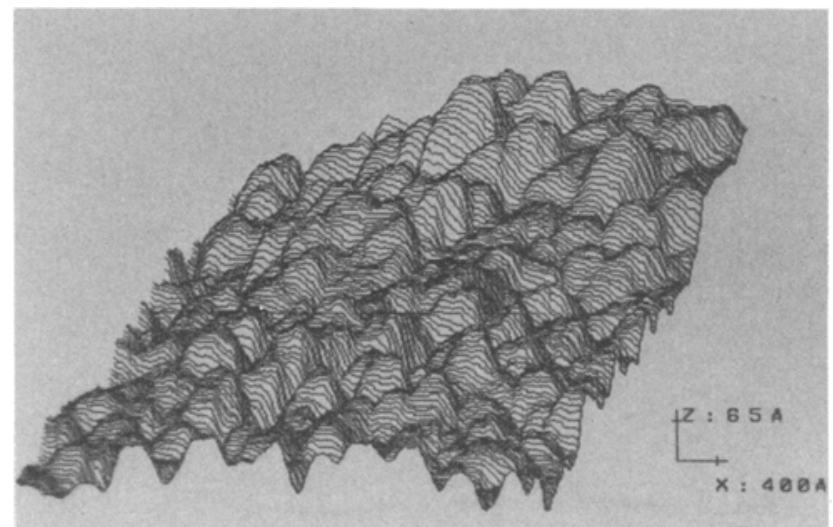

Fig. 6. (a, left) STM image of electrodispersed Pt overlayer obtained immediately after its formation; (b, right) the same specimen after $6 \times 10^{4} \mathrm{~s}$ aging in $\mathrm{H}_{2} \mathrm{SO}_{4} 325 \mathrm{~K}$. Total area imaged is $0.62 \times 0.62 \mu \mathrm{m}$. 
When Eq. [10] is written as follows

$$
\frac{1}{R}=\left[3\left(\frac{2 \gamma a^{4} D_{\mathrm{s}}}{k T}\right)^{1 / 4} \frac{(z \mathbf{F} \rho)}{\pi M q}\right] t^{1 / 4}
$$

The plot $1 / R v s . t^{1 / 4}$ yields straight lines for the interval of $t$ compatible with the validity of Eq. [4]. From the value of $B$, the slope of the straight lines which is given by the equation

$$
B=3\left(\frac{2 \gamma \alpha^{4} D_{s}}{k T}\right)^{1 / 4}\left(\frac{z \mathrm{p} \mathbf{F}}{\pi M q}\right)
$$

it is possible to evaluate $D_{\mathrm{s}}$ by using, $\gamma=900$ and $\gamma=1800$ $\mathrm{erg} / \mathrm{cm}^{2}$ and $a=4.07 \times 10^{-8}$ and $3.9 \times 10^{-8} \mathrm{~cm}$ for $\mathrm{Au}$ and $\mathrm{Pt}$, respectively (18) and the $q$ values resulting from the electrochemical measurements. The $D_{\mathrm{s}}$ values obtained for both metals are presented in a ln $D_{\mathrm{s}}$ vs. 1/T plot in Fig. 7 .

As expected from Eq. [11] and [12] the slope B decreases linearly as $q$ and $R$ increase (Fig. 8). When condition [3] is fulfilled, as it is for the case of $T=298 \mathrm{~K}$ and $t>300 \mathrm{~s}$, the value of $r$ must be smaller than 10 and $116 \AA$ for $\mathrm{Pt}$ and $\mathrm{Au}$, respectively. These figures, however, are certainly lower than those directly obtained from STM-SEM imaging, due to the proper coalescence process interfering during measurements.

On the other hand, at the temperature $T$, the value of $D_{\mathrm{s}}$ depends on the $T / T m$ ratio, where $T m$ is the melting temperature. It should be noted that for each metal $D_{\text {s }}$ increases as $T$ increases, and that for a constant $T, D_{\mathrm{s}}$ for Pt is lower than that for Au (Fig. 7). Furthermore, the plot of data $\ln D_{\mathrm{s}}$ vs. $1 / T$ can be reasonably fitted to straight lines, the slope of these lines yielding an activation energy for surface diffusion, $E a$, close to 14 and $19 \mathrm{kcal} / \mathrm{mol}$ for $\mathrm{Au}$ and $\mathrm{Pt}$, respectively. Both $\mathrm{E} a$ values are lower than those reported in the literature for surface diffusion of $\mathrm{Au}$

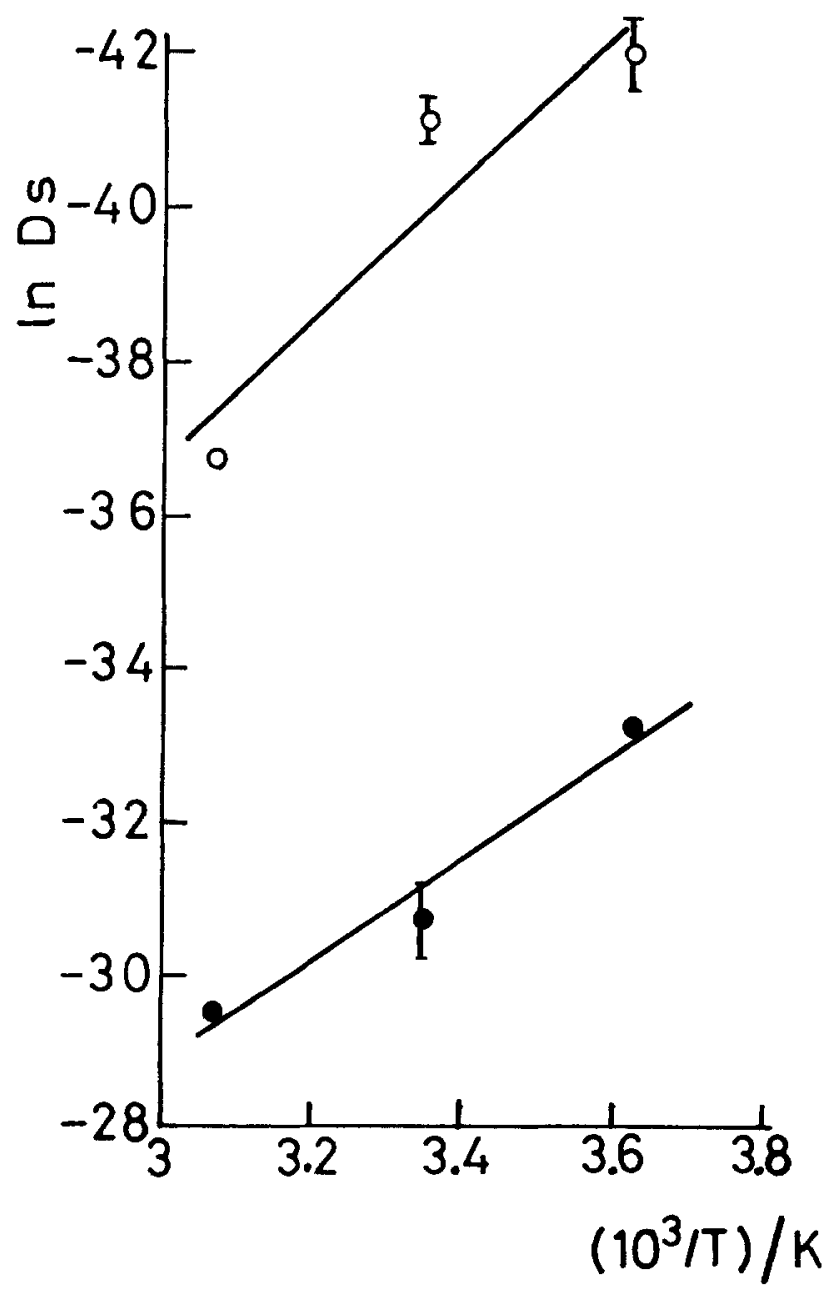

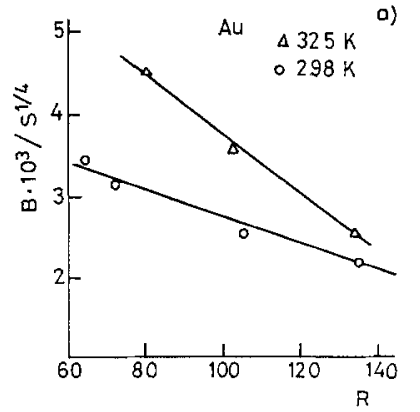
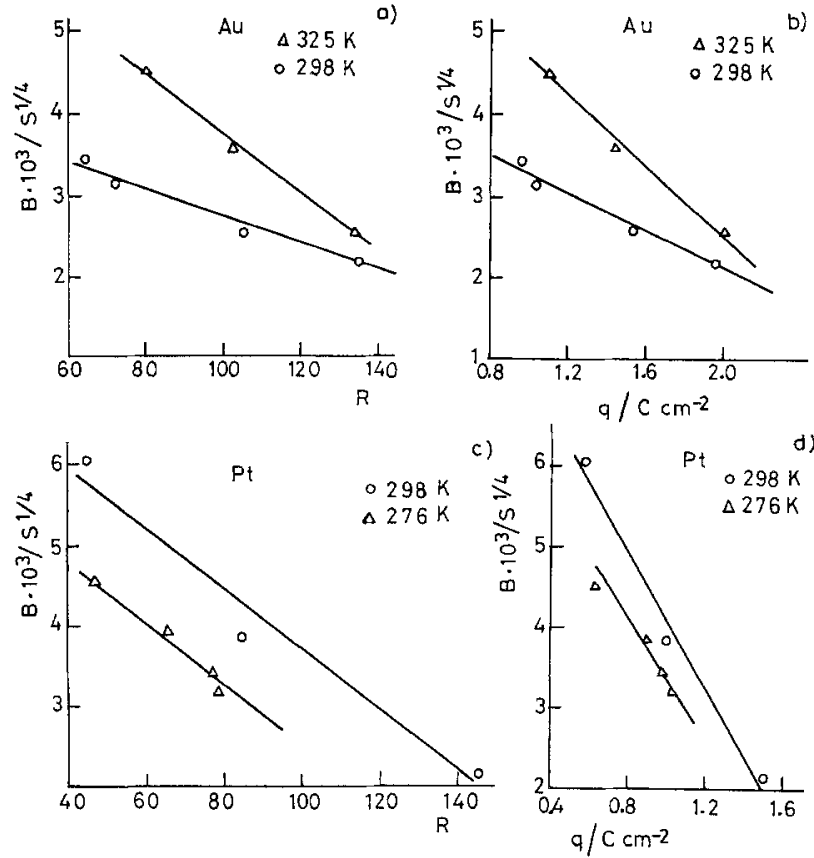

Fig. 8. $R$ vs. $B$ (a) and $q$ vs. $B$ (b) plots for electrodispersed Au overlayers; $R$ vs. $B$ (c) and $q$ v5. $B$ (d) plots for electrodispersed Pt overlayers.

[10-40 kcal/mol (1); $21 \mathrm{kcal} / \mathrm{mol}(19)]$ and Pt [22 kcal $/ \mathrm{mol}(1)]$ in vacuum and in air. Therefore, the $D_{\mathrm{s}}$ values for atomically flat $\mathrm{Au}$ in vacuum at room temperature obtained by STM measurements was estimated between $10^{-16}$ and $10^{-20}$ $\mathrm{cm}^{2} \mathrm{~s}^{-1}(20)$. A comparison between recent data for $\mathrm{Au}$ in air obtained by peak to peak measurements using STM (19) and our results are also presented in Fig. 9. The differences are not surprising when one takes into account the gap between surface processes in vacuum and at condensed interfaces. The increased surface mobility as compared to the metal/vacuum system can be related to anions and $\mathrm{H}_{2} \mathrm{O}$ molecule adsorption on the metal surfaces. This fact correlates very well with the recent results obtained by in situ STM microscopy $(21,22)$ which show that adsorbed ions like $\mathrm{Cl}^{-}$increase the surface atomic mobility.

\section{Conclusions}

For the first time, a simple method has been developed for measuring surface diffusion coefficients in electrochemical systems involving electrodispersed $\mathrm{Au}$ and $\mathrm{Pt}$ electrodes. The method is based on the time dependence of the surface roughness factor of metal overlayers. The method requires a model of the rough metal structure in order to obtain the relation between the surface roughness factor and the geometric parameters of the electrodispersed metal layer. This model is based on the measure-

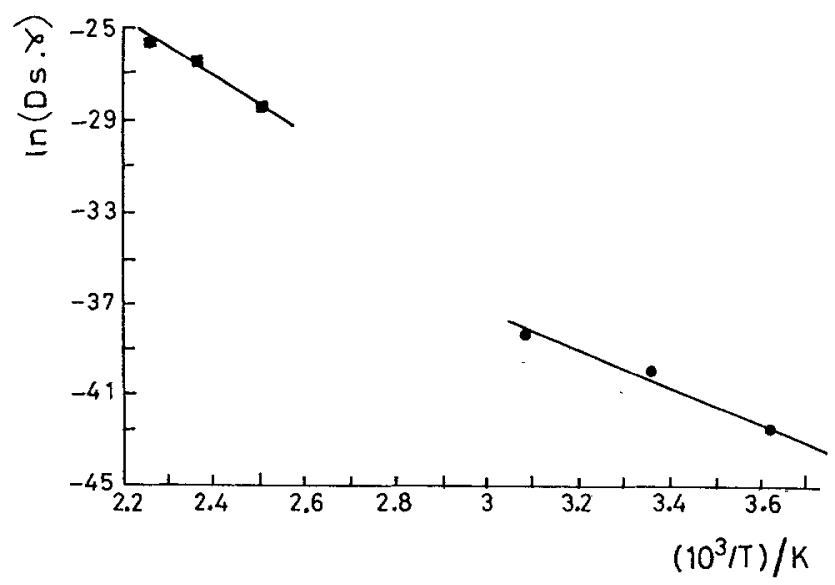

Fig. 9. In $D_{s} \gamma$ vs. $1 / T$ plots for $A u$ in air $[\operatorname{Ref.~(19)]~(\square )~and~for~} A u$ in 
ment of the surface topography of the electrodes by STM in particular the size and diameter of the columnar-like structure and its evolution during time. It is shown that, for a rounded cap columnar structure, the estimation of the thickness by coulometry and the evaluation of the roughness factor through conventional voltammetry allow one to obtain the value of the surface diffusion coefficient. For $\mathrm{Au}$ and $\mathrm{Pt}$ the measured values of the surface diffusion coefficients in $0.5 \mathrm{M} \mathrm{H}_{2} \mathrm{SO}_{4}$ appear to be higher than those reported in vacuum.

\section{Acknowledgment}

A fellowship from the Consejo Nacional de Investigaciones Cientificas y Técnicas (Argentina) to R.C.S. is gratefully acknowledged. Financial support was obtained from DGICT through contract number PB86-0606. We are indebted to A. Buendia for skillful technical assistance and to J. Gómez and J. M. Gómez for help in data acquisition and images processing.

Manuscript submitted April 18, 1989; revised manuscript received ca. Jan. 15, 1990.

Universidad Autonoma de Madrid assisted in meeting the publication costs of this article.

\section{REFERENCES}

1. H. P. Bonzel, in "Surface Physics of Materials," J. M. Blakely, Editor, p. 280, Academic Press, Inc., New York (1975).

2. G. E. Rhead, Surf. Sci., 192, 597 (1987).

3. M. Z. Allmang and L. C. Feldman, Appl. Surf. Sci., 33/34, 395 (1988).
4. W. W. Mullins, J. Appl. Phys., 30, 77 (1959).

5. B. K. Chakraverty, J. Phys. Chem. Solids, 28, 2401 (1967).

6. A. D. Abramenkov, V. V. Slezov, L. Tanatarov, and Y. M. Fogel, Fiz. Tvevd. Tela, 12, 2934 (1971).

7. L. Vázquez, A. Bartolomé, A. Baró, C. Alonso, R. C. Salvarezza, and A. J. Arvia, Surf. Sci., 215, 171 (1989).

8. T. Hepel, This Journal, 134, 2685 (1987).

9. A. Honji, T. Mori, K. Tamura, and Y. Hishinuma, ibid., 135, 355 (1988)

10. A. J. Arvia, R. C. Salvarezza, and W. E. Triaca, Electrochim. Acta, In press.

11. J. R. Galvele, This Journal, 133, 953 (1986).

12. A. C. Chialvo, W. E. Triaca, and A. J. Arvia, J. Electroanal. Chem., 171, 303 (1984).

13. M. E. Vela, R. C. Salvarezza, and A. J. Arvia, Electrochim. Acta, In press.

14. A. E. Bolzan, A. M. Castro Luna, A. Visintin, R. C. Salvarezza, and A. J. Arvia, ibid., 33, 1743 (1988).

15. A. C. Chialvo, W. E. Triaca, and A. J. Arvia, J. Electroanal. Chem., 146, 93 (1983); 171, 303 (1984).

16. L. Vázquez, A. Bartolomé, R. Garcia, A. Buendía, and A. M. Baró, Rev. Sci. Instrum., 59, 1286 (1988).

17. A. Bartolomé, R. García, L. Vázquez, and A. Baró, J. Microscopy, 152, 205 (1988).

18. "Handbook of Chemistry and Physics," R. C. Weast, Editor, CRC Press Inc., Boca Raton FL (1979).

19. T. Lin and Y. Chung, Surf. Sci., 207, 539 (1989).

20. J. Schneir, R. Sonnenfeld, O. Marti, P. K. Hansma, J. E. Demuth, and J. Hamers, J. Appl. Phys., 63, 717 (1988).

21. J. Wiechers, T. Twomey, D. M. Kolb, and R. J. Behm, J. Electroanal. Chem., 248, 451 (1988).

22. D. J. Trevor, C. E. D. Chidsey, and D. N. Loiacono, Phys. Rev. Lett., 62, 929 (1989).

\title{
The Electrocatalytic Reduction of Nitrate Mediated by Underpotential-Deposited Cadmium on Gold and Silver Electrodes in Acid Media
}

\author{
Xuekun Xing* and Daniel A. Scherson* * \\ Case Center for Electrochemical Sciences and The Department of Chemistry, Case Western Reserve University, \\ Cleveland, Ohio 44106 \\ Cecilia Mak ${ }^{\star \star}$ \\ AT\&T Bell Laboratories, Murray Hill, New Jersey 07974-2070
}

\section{ABSTRACT}

Rotating ring-disk electrode techniques have been employed for the investigation of the electrocatalytic reduction of nitrate induced by underpotential-deposited cadmium on Au and Ag surfaces. Based on Albery's kinetic analysis it has been concluded that for concentrations of nitrate in the millimolar range, the reaction products depend on the $p H$ of the solution. Specifically, for $p \mathrm{H}=3$, the reaction yields predominantly nitrite, whereas for $\mathrm{pH}=1$, the process proceeds beyond the nitrite stage to generate products which do not undergo oxidation on a Au ring electrode polarized at potentials as high as $1.0 \mathrm{~V} v s$. SCE. Additional evidence in support of these results was provided by exhaustive bulk electrolysis experiments. The lack of linearity of some of Albery's diagnostic plots could be accounted for quantitatively by a numerical integration of the differential equations which govern disproportionation-type reactions at rotating ring electrodes. Good agreement was found between theory and experiment using independently determined values for the rate constant of disproportionation of $\mathrm{NO}_{2}$ in solution.

The reduction of nitrate ions has received considerable attention over the last three decades, mainly because of the possibility of utilizing abundant and inexpensive sources for the production of useful chemicals (1-9). Examples of these include $\mathrm{N}_{2} \mathrm{O}$, a compound used as an anesthetic in medical applications, $\mathrm{NH}_{3}$, a nitrogen source in fertilizers, and hydroxylamine, $\mathrm{NH}_{2} \mathrm{OH}$, a material of crucial importance in the manufacture of caprolactam and aldoximes. Hydroxylamine undergoes protonation in nitric acid to yield hydroxylammonium nitrate which is an effi-

* Electrochemical Society Student Member.

** Electrochemical Society Active Member.

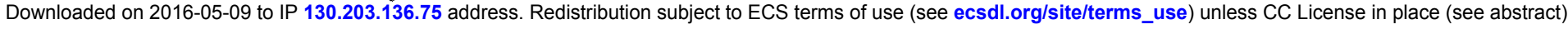

cient agent for the reduction of plutonium to the trivalent state and a key ingredient in the development of liquid propellant systems (10). An area in which the conversion of nitrate into ammonia may also find application is the recycling of strongly alkaline solutions used in the neutralization of nitric acid waste clean-up solutions in the nuclear industry (11).

The large scale production of some of the reduced forms of nitrate has involved almost exclusively a combination of homogeneous phase reactions and simple heterogeneous catalysis (12-14). The possibility of using electrochemical techniques for these processes appears espe- 